\title{
Financement et industrie en Mauricie, 1900-1950
}

\section{Claude Bellavance, Normand Brouillette et Pierre Lanthier}

Volume 40, numéro 1, été 1986

URI : https://id.erudit.org/iderudit/304423ar
DOI : https://doi.org/10.7202/304423ar

Aller au sommaire du numéro

\section{Éditeur(s)}

Institut d'histoire de l'Amérique française

\section{ISSN}

0035-2357 (imprimé)

1492-1383 (numérique)

\section{Découvrir la revue}

\section{Citer cet article}

Bellavance, C., Brouillette, N. \& Lanthier, P. (1986). Financement et industrie en Mauricie, 1900-1950. Revue d'histoire de l'Amérique française, 40(1), 29-50. https://doi.org/10.7202/304423ar

\section{Résumé de l'article}

Posant l'hypothèse que des changements dans le financement des grandes entreprises peuvent contribuer à la stagnation industrielle des régions périphériques ou semi-périphériques où elles se sont développées, les auteurs étudient l'évolution financière de trois groupes industriels qui ont exercé un rôle prédominant dans le développement de la Mauricie avant 1950. Après une brève présentation du contexte économique et technologique dans lequel s'inscrit l'essor industriel mauricien, ainsi que des causes déjà connues du plafonnement survenu à la fin des années cinquante, suit un examen détaillé des stratégies de financement de chacun des groupes. A l'analyse, surgissent des différences qui tiennent à la nature, à la taille et à la maturité des firmes. Au-delà de cette diversité, se dégage une similitude de politique selon les périodes considérées : ainsi, les liaisons personnelles entre les entrepreneurs et les investisseurs furent déterminantes initialement ; durant les années 1910 et 1920, les sources d'investissement se dépersonnalisèrent tant par l'augmentation du nombre d'actionnaires que par la présence accrue d'institutions financières ; la crise des années 1930, bloquant le financement extérieur, incita le recours à des pratiques amplifiant l'autonomie financière des firmes. Cette évolution n'est cependant pas étrangère au processus de maturation des sociétés qui apparaît en définitive comme un des facteurs décisifs de l'évolution industrielle de la Mauricie. Ce n'est donc qu'indirectement que les stratégies financières auront pu provoquer le blocage industriel de la région. Dans cette perspective, la méfiance, si elle existait, des milieux d'affaires canadiens à l'endroit de l'industrie, de même que les motivations de pouvoir des investisseurs prennent peu d'importance comme facteurs explicatifs.
Tous droits réservés @ Institut d'histoire de l'Amérique française, 1986
Ce document est protégé par la loi sur le droit d'auteur. L'utilisation des services d'Érudit (y compris la reproduction) est assujettie à sa politique d'utilisation que vous pouvez consulter en ligne.

https://apropos.erudit.org/fr/usagers/politique-dutilisation/ 


\title{
FINANCEMENT ET INDUSTRIE EN MAURICIE, 1900-1950
}

\author{
CLAUDE BELLAVANCE ${ }^{1}$ \\ NORMAND BROUILLETTE \\ PIERRE LANTHIER \\ Études québécoises \\ Université du Québec à Trois-Rivières
}

\section{RÉSUMÉ}

Posant l'hypothèse que des changements dans le financement des grandes entreprises peuvent contribuer à la stagnation industrielle des régions périphériques ou semi-périphériques où elles se sont développées, les auteurs étudient l'évolution financière de trois groupes industriels qui ont exercé un rôle prédominant dans le développement de la Mauricie avant 1950. Après une brève présentation du contexte économique et technologique dans lequel s'inscrit l'essor industriel mauricien, ainsi que des causes déjà connues du plafonnement survenu à la fin des années cinquante, suit un examen détaillé des stratégies de financement de chacun des groupes. A l'analyse, surgissent des différences qui tiennent à la nature, à la taille et à la maturité des firmes. Au-delà de cette diversité, se dégage une similitude de politique selon les périodes considérées: ainsi, les liaisons personnelles entre les entrepreneurs et les investisseurs furent déterminantes initialement; durant les années 1910 et 1920, les sources d'investissement se dépersonnalisèrent tant par l'augmentation du nombre d'actionnaires que par la présence accrue d'institutions financières; la crise des années 1930, bloquant le financement extérieur, incita le recours à des pratiques amplifiant l'autonomie financière des firmes. Cette évolution n'est cependant pas étrangère au processus de maturation des sociétés qui apparaît en définitive comme un des facteurs décisifs de l'évolution industrielle de la Mauricie. Ce n'est donc qu'indirectement que les stratégies financières auront pu provoquer le blocage industriel de la région. Dans cette

1 Claude Bellavance est actuellement étudiant au doctorat à l'Université du Québec à Montréal. Les auteurs remercient sincèrement René Hardy, Normand Séguin et Roger Héroux pour leurs commentaires sur ce texte. Ils tiennent également à souligner le support financier du Conseil de recherche en sciences humaines du Canada. 
perspective, la méfiance, si elle existait, des milieux d'affaires canadiens à l'endroit de l'industrie, de même que les motivations de pouvoir des investisseurs prennent peu d'importance comme facteurs explicatifs.

\section{ABSTRACT}

Putting forward the hypothesis that changes in large firms' financing strategies may contribute to the industrial stagnation of peripheral or semi-peripheral regions where these firms have developed, the authors study the financial evolution of three industrial groups which have played a predominant role in the development of the Mauricie before 1950. After a short presentation of the economic and technological framework in which the region's economic growth has taken place, and of the already known reasons for the slowdown of the regional economy at the end of the 1950s, they closely examine each group's financing strategies. The analysis reveals differences which stem mainly from the nature, size and maturity of the firms. Beyond this diversity, and for each period considered, a certain similarity in the policies can be perceived: in the beginning, for instance, personal contacts between the entrepreneurs and the main investors were decisive; during the 1910s and 1920s, the sources of investment became more impersonal with the increase in the number of shareholders and with the growing presence of financial institutions; during the Depression, external financing almost stopped, and firms relied on more autonomous financing. The maturation process of the firms explains in large part this evolution, and appears to be one of the main factors of the Mauricie's industrial evolution. Therefore, financial strategies contributed only indirectly to the industrial slowdown of the region. In this perspective, Canadian business circles' mistrust of industry, if it existed, just as the investors' power motivation, appear to be far less important as explaining factors.

Jusqu'en 1950, le développement de la Mauricie s'identifiait à toutes fins utiles à celui de la grande industrie. Les liens entre la région et les entreprises industrielles paraissaient même indissolubles. Et pourtant, à partir des années cinquante, ce ne fut plus le cas: les grandes sociétés boudèrent la Mauricie. Même celles déjà implantées dans le milieu préférèrent poursuivre leur croissance ailleurs. Diverses explications à ce phénomène furent proposées, allant de l'évolution technologique jusqu'au renouvellement du patronat. Mais, au fur et à mesure que se sont approfondies les recherches sur cette question, force a été d'admettre qu'il n'existe pas de réponse simple et qu'il est préférable d'élargir le plus possible le champ d'investigation. Dans cette perspective, l'étude du financement des entreprises pourrait-elle ajouter une 
pierre à l'édifice? De récentes recherches ont montré, entre autres choses, qu'il existait au 19e siècle un problème structurel de développement des industries rurales en Mauricie lié aux difficultés de financement ${ }^{2}$. L'absence d'un marché local important favorisait peu l'implantation d'institutions financières. Quelles furent les solutions adoptées par les dirigeants des grandes entreprises au début du 20e siècle? Et plus tard, la crise des années trente aurait-elle suscité de nouvelles difficultés financières compromettant tout développement industriel ultérieur en milieu semi-périphérique?

A défaut d'une source fiable à l'échelle régionale et d'une mesure exacte du mouvement des capitaux en Mauricie, il a fallu se reporter sur un choix d'entreprises qui puissent à la fois être suffisamment représentatives de la région et posséder une comptabilité ne posant pas trop de problèmes d'interprétation. Ont été retenues les entreprises suivantes: un groupe s'occupant d'électricité et de chimie, la Shawinigan Water \& Power (SWPC); une entreprise de textile, la Wabasso Cotton et deux de ses filiales, la Shawinigan Cotton et la St.Maurice Valley Cotton; enfin, une société de pâtes et papiers, la Consolidated Paper Ltd et deux de ses ancêtres, la Laurentide Co Ltd et la Wayagamack Pulp \& Paper Ltd.

Certaines précisions s'imposent sur l'approche retenue. Souvent, les études de financement s'attachent à l'investisseur, au fournisseur de capitaux. Dans cette perspective, l'évolution conjoncturelle des marchés financiers ainsi que les rapports de pouvoir prennent le premier plan. Notre démarche privilégiera plutôt le point de vue de l'industriel, de celui qui gère l'entreprise. Selon cette optique, les problèmes de conjoncture et de relations de pouvoir sont certes bien importants, mais ils sont dilués dans le souci beaucoup plus immédiat d'équilibrer constamment les besoins suscités par la marche de l'entreprise et les ressources financières à sa disposition. Les priorités ne sont donc pas les mêmes. Supposons, par exemple, qu'il faille choisir un mode de financement. Pour l'investisseur, le choix se borne aux diverses catégories de titres que la société offre. Pour l'entrepreneur, l'éventail s'élargit: en plus des titres, il doit se préoccuper de la capacité de la société à s'autofinancer, débattre les mérites respectifs des dettes à long terme et de celles à courte échéance, s'enquérir de la solvabilité de ses clients et de ses fournisseurs, etc. Notre étude ne mettra pas au premier plan les effets de la conjoncture financière nationale et internationale. Il va de soi cependant que cette conjoncture a insufflé son rythme aux grands mouvements d'investissement, en région comme ailleurs. Ainsi, les effets des crises des débuts des années vingt et des années trente sont

\footnotetext{
2 Sur cette question, on consultera la communication de R. Hardy, P. Lanthier et N. Séguin portant sur l'industrie rurale dans le comté de Champlain (Qué.) au 19e siècle, donnée en septembre 1985 dans le cadre du Colloque franco-québécois sur les relations ville-campagne (à paraître en 1986).
} 
indéniables. De même, la vague de concentration et de renouvellement d'équipements des années 1926-1929 fut grandement facilitée par la forte croissance économique ayant caractérisé cette période. Ce sont les stratégies financières en tant que produits des rapports entre les ressources et les besoins de la grande entreprise dont il sera question ici. Nous tenterons plus précisément de comprendre de quelle manière la nature des activités des firmes et le caractère semi-périphérique de l'espace dans lequel s'inséraient ces activités ont modelé les stratégies financières des groupes industriels étudiés.

S'intéresser au financement de la grande entreprise industrielle nous entraîne à employer certains concepts et à évoquer des pratiques spécifiques. Il convient, avant d'entrer dans l'analyse, d'apporter des éclaircissements sur quelques-uns d'entre eux. Ainsi, bien que financement et investissement soient les deux faces d'une même réalité, il est nécessaire de les distinguer. Par investissement, nous entendons la mobilisation et l'utilisation de capitaux. Le financement renvoie quant à lui à la provenance de ces capitaux. Quand une entreprise désire faire de nouveaux investissements, elle doit d'abord trouver une source de financement. Quatre possibilités s'offrent alors: émissions d'actions, emprunts à long terme (obligations), autofinancement (par profits non distribués ou par remboursement d'emprunts), et emprunts à court terme (prêt bancaire par exemple). Le choix entre ces possibilités sera fonction non seulement de la performance économique de l'entreprise ou de l'état du marché financier, mais aussi du type d'investissement à faire. Par exemple, une firme désirant se doter de nouveaux équipements ou, plus généralement, accroître substantiellement ses immobilisations, aura le plus souvent recours aux emprunts à long terme (obligations, debenture, etc.) ou à un accroissement de son capital-actions. Et s'il s'avère nécessaire de disposer temporairement d'un surplus de liquidités, entre autres choses, pour rembourser rapidement une dette, elle pourra s'adresser à une institution bancaire et demander un prêt à court terme.

Ce texte, dans un premier temps, présentera le contexte économique et technologique particulier dans lequel s'inscrit le développement industriel mauricien, pour ensuite rendre compte des causes du plafonnement survenu dans les années cinquante. Suivra, dans un second temps, l'étude financière en tant que telle: après une analyse consacrée aux investissements, nous serons en mesure de dégager les similitudes de comportement grâce à l'observation cas par cas des groupes retenus. Un regroupement diachronique des grandes tendances du financement de ces entreprises terminera cet article.

\section{A - L'espace industriel mauricien: une mise en contexte}

On ne saurait comprendre le développement de l'espace industriel mauricien et plus généralement de l'hinterland québécois au 20e siècle 
sans faire référence au contexte économique et technologique dans lequel il s'inscrit. Au début du siècle, moment où la Mauricie s'ouvre véritablement à l'industrialisation, d'importants progrès techniques avaient été réalisés. Ainsi, l'inauguration de la centrale de Niagara Falls aux États-Unis en 1896 lança l'ère industrielle de l'hydro-électricité. Grâce aux efforts de la recherche scientifique, cette curiosité de laboratoire qu'était l'aluminium put être produite sur une grande échelle et à des coûts nettement avantageux. Dans le domaine des pâtes et papiers, un certain nombre d'innovations tout au long du $19 \mathrm{e}$ siècle rendirent possibles la transformation du bois en papier et l'avènement de la fabrication en continu. Bref, à la fin du siècle dernier, un ensemble de procédés techniques nouveaux vinrent bouleverser le paysage industriel. Combinés au renversement de la tendance à la baisse des prix en 1896, qui stimula vigoureusement la propension à l'investissement, ils permirent l'apparition d'activités industrielles basées sur l'exploitation des richesses naturelles, ce qui entraîna, dans un laps de temps relativement court, des modifications d'envergure à la structure des régions bien dotées en ressources hydrauliques et forestières.

Dans ce contexte économique et technologique, la vallée du SaintMaurice apparut rapidement comme un territoire plein de promesses. Jouissant d'un potentiel hydro-électrique et forestier assez exceptionnel, reliée dès le début au réseau ferroviaire nord-américain, elle allait connaître au cours de la première moitié du siècle une croissance industrielle tout à fait remarquable. Ainsi, alors que pour l'ensemble du Québec l'indice de la valeur des productions manufacturières $(1900=$ 100) atteignait 3100 en 1951, il excédait les 6000 points pour la Mauricie ${ }^{3}$.

Au plan spatial, la structure industrielle s'articule autour de l'axe du Saint-Maurice, véritable épine dorsale du développement économique régional. Aux concentrations urbaines et industrielles des agglomérations de Shawinigan, Grand-Mère et Trois-Rivières s'oppose un arrière-pays agricole restreint, peu peuplé, qui prend fin rapidement au nord avec les grandes solitudes du Bouclier canadien.

De nombreuses caractéristiques de l'industrie mauricienne l'apparentent à celle d'autres régions de l'hinterland québécois. Tout d'abord, dans une très large proportion, elle est fondée sur les ressources naturelles; elle a donné lieu en effet à la mise en place d'une base industrielle relativement étroite, axée sur les pâtes et papiers, l'électrochimie, l'électrométallurgie et à un degré moindre sur les textiles et le vêtement. On constate également une extraversion très prononcée des industries régionales, le gros des productions étant ache-

\footnotetext{
3 N. Brouillette, Le développement industriel d' une région du proche hinterland québécois: la Mauricie, 1900-1975, Thèse de doctorat, Université McGill, 1983, 137.
} 
miné vers l'étranger ou à tout le moins vers les grands centres nationaux. Signalons enfin un dernier trait: la grande taille des centrales hydro-électriques et des établissements manufacturiers érigés à proximité. La rentabilité des activités passait par des volumes de production importants pouvant seuls permettre des économies d'échelle appréciables et, partant, une rémunération suffisante du capital.

Après la reprise des années quarante où elle a confirmé sa suprématie sur les autres régions de l'hinterland québécois ${ }^{4}$, la Mauricie entra dans une période de ralentissement de la croissance, voire de déclin, qui allait se poursuivre jusqu'à nos jours. L'arrivée de la Canadian Westinghouse à Trois-Rivières, en 1951, mit fin en pratique à la longue suite d'implantations majeures qui avaient placé la vallée du SaintMaurice au premier rang sur l'échiquier industriel de l'hinterland québécois.

Bon nombre de causes ont été mises de l'avant pour expliquer le blocage que connaît la Mauricie industrielle depuis maintenant près de trois décennies ${ }^{5}$. Ainsi, il ne fait pas de doute que les avantages comparatifs dont elle bénéficiait dans les trente premières années du siècle se sont fortement amoindris: l'épuisement des ressources forestières locales et la force d'attraction désormais négligeable des centrales hydroélectriques pour les industries grandes consommatrices d'énergie expliquent dans une large mesure ce plafonnement. Par ailleurs, un vaste mouvement de restructuration des espaces industriels traverse les sociétés occidentales depuis 1945, résultant en une relocalisation de la fabrication des produits de base et des produits semi-finis. La Mauricie, qui s'était fait une spécialité de ces productions, ne pouvait être que perdante à long terme. En outre, les stratégies de croissance de bon nombre des grandes firmes présentes en Mauricie allaient dans le sens de la diversification et de l'intégration verticale vers le produit fini, et donc vers une localisation des nouveaux établissements manufacturiers à proximité des grands marchés. Elles ont détourné de la région les investissements récents de ces firmes. Enfin, à partir des années quarante, l'arrivée aux postes de commande d'une nouvelle génération de dirigeants, moins préoccupée du développement des établissements régionaux que de celui de l'ensemble de la firme, a probablement contribué à sa manière au ralentissement de la croissance mauricienne.

L'autopsie de la stagnation industrielle de la région fait donc appel à des causes nombreuses, variées. Jusqu'à ce jour cependant, on n'a guère considéré les aspects strictement financiers du problème.

4 N. Brouillette, op. cit., 83.

Voir à ce sujet, N. Brouillette, op. cit., 250-294. P. Lanthier, «Stratégie industrielle et développement régional: le cas de la Mauricie au XXe siècle», in Revue d'histoire de l'Amérique française, 37,1 (juin 1983): 3-19. C. Bellavance, «Patronat et entreprise au 20e siècle: l'exemple mauricien», in Revue d' histoire de l'Amérique française, 38,2 (automne 1984): 181-201. 
Figure 1

Evolution décennale des nouveaux investissements de trois groupes industriels en Mauricie, en millions de $\$$ constants 1898-1950

WABASSO COTTON

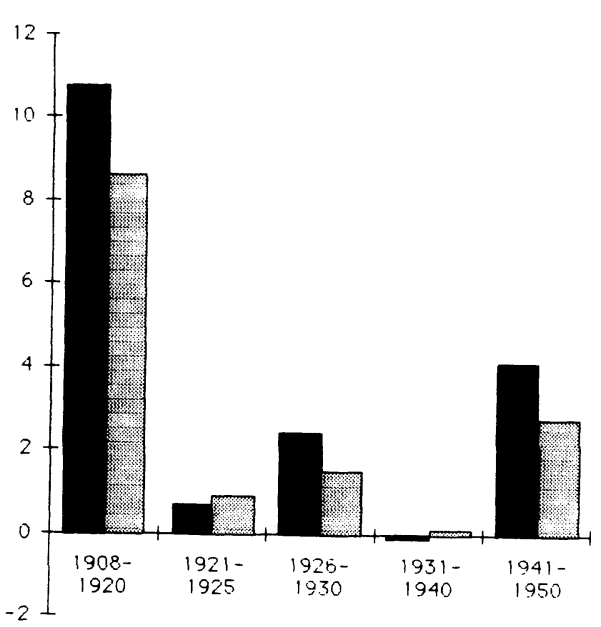

Note: dans le cas de la Laurentide, il n'a pas eté tenu compte des Source: rapports annuels des entreprises, annees concernees

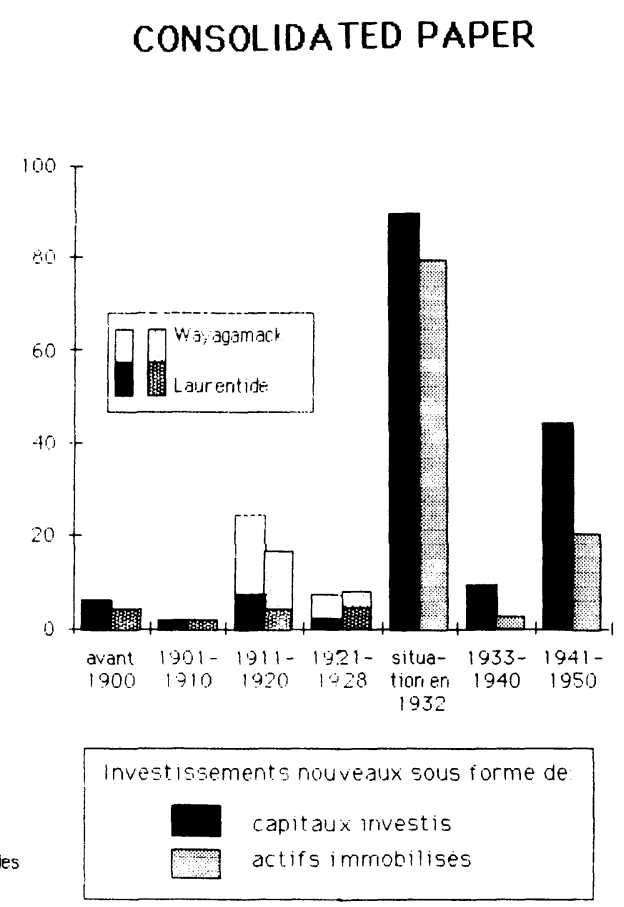

SHAWINIGAN WATER \& POWER

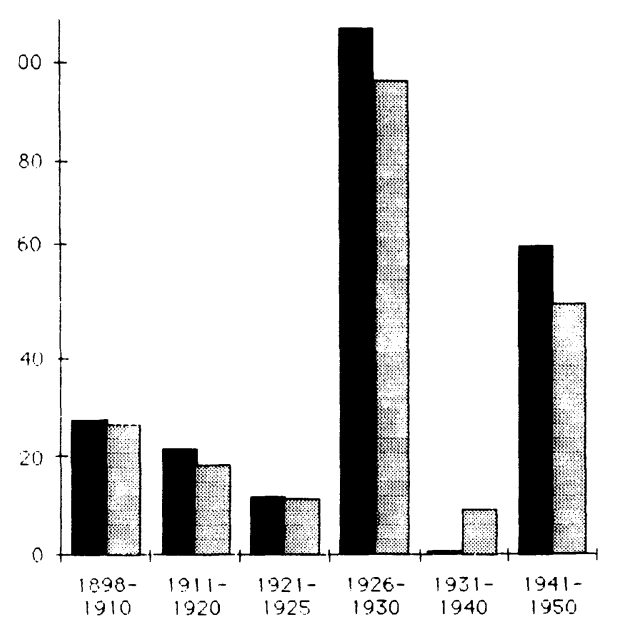

$\stackrel{w}{u}$ 


\section{$B$ - Étude financière des trois sociétés}

Les trois firmes témoins, la SWPC, la Consolidated Paper et la Wabasso ont été responsables d'une très large part de l'investissement industriel régional au cours de la période étudiée. Ceci nous incite donc à examiner brièvement le développement de leurs investissements qui d'ailleurs se réalisent surtout en Mauricie au cours de ces années.

La figure 1 résume, par tranches décennales, la valeur totale des investissements nouveaux, en dollars constants (base 1935-1939 = 100). Sur le plan matériel, ces investissements correspondaient pour toutes les sociétés, d'une part à la construction d'usines ou de centrales, de l'autre à l'acquisition d'un fonds de roulement, en particulier pour la Wabasso et pour la Consolidated Paper. Ces investissements sont exprimés sous forme de capitaux investis et d'actifs immobilisés. Il convient de définir ici ces deux notions: la première réfère à la totalité des actifs d'une société, qu'ils soient immobiles ou circulants, alors que la seconde concerne uniquement la partie fixe des investissements. La lecture de la figure peut donner au lecteur l'impression que, dans une même décennie, les actifs immobilisés sont supérieurs aux capitaux investis. On le voit bien dans le cas de la SWPC en 1931-1940. Ce paradoxe apparent s'explique par le fait que les actifs immobilisés nouveaux de cette décennie ont été prélevés dans les actifs circulants déjà existants. L'absorption des actifs d'autres sociétés a également été retenue dans cette figure. Ce fut le cas, notamment de la SWPC en 1928 et, de 1926 à 1931, du regroupement des usines de la Laurentide (GrandMère), de la Belgo (Shawinigan), de la St.Maurice Paper (Cap-de-laMadeleine) et de la Wayagamack (Trois-Rivières) avec celle de Port Alfred, pour former, après maintes péripéties, la Consolidated Paper. Les résultats de ce regroupement sont rassemblés sur la figure dans «situation en 1932». Ce processus nécessita un appel considérable de capitaux, mais peu d'investissement matériel.

A l'examen, l'évolution sur toute la période considérée se révèle fort complexe. La poussée des premières années ne s'accomplit pas de manière brusque, mais graduelle, sauf dans les cas de la Wabasso et de la Wayagamack, dirigées en partie par les mêmes individus et de création plus récente. Orı notera cependant, dans le cas des ancêtres de la Consolidated Paper, que la figure ne reprend que deux sociétés sur cinq, ce qui sous-estime fortement l'importance des investissements avant 1925 . Les années vingt, surtout celles précédant immédiatement la crise, amenèrent une croissance considérable, destinée tant à l'extension ou au renouvellement des immobilisations qu'à un regroupement de sociétés. Bien entendu, la crise freina ce processus. Toutefois la guerre et plus encore l'après-guerre permirent une reprise. Son amplitude cependant fut limitée. Seule la SWPC connut une expansion significative. Mais elle concernait surtout la construction de centrales et ne 
générait pas à vrai dire d'emploi industriel. Pour leur part, la modernisation de la Consolidated Paper et l'érection à Grand-Mère d'une usine de la Wabasso constituaient de bien faibles investissements comparés à ceux de l'avant-crise. L'origine des capitaux serait-elle en cause? Une étude du financement de chaque groupe permettra d'avancer quelques réponses.

\section{La Shawinigan Water and Power 6}

La SWPC a été fondée en 1898 par un groupe d'hommes d'affaires américains alliés à quelques membres influents de la grande bourgeoisie montréalaise. La compagnie entreprit dès l'année 1899 la construction à Shawinigan de ce qui allait devenir l'une des plus grandes centrales hydro-électriques de l'époque. L'aménagement des chutes de Shawinigan a posé des défis considérables: recours à une technologie de pointe encore peu éprouvée, difficulté d'ériger une centrale loin des grandes villes, voies de communication déficientes, etc. Mais les problèmes de financement étaient plus graves encore.

Au cours de ces premières années, la situation était d'autant plus difficile que l'immense majorité des actions émises lors de la formation de la compagnie ne furent jamais véritablement souscrites. Elles avaient presque toutes été attribuées à J. Joyce en échange de ses droits sur les chutes de Shawinigan. En fait, la principale source de financement venait d'emprunts à long terme garantis par l'hypothèque de ce qui avait déjà été construit. La survie même de la compagnie d'électricité ne devint possible que grâce à l'appui de financiers prestigieux qui s'en portèrent maintes fois garants. La jeune compagnie n'avait décroché que quelques contrats de fourniture d'énergie hydraulique lorsque J. E. Aldred se rendit à New York pour rencontrer des bailleurs de fonds. Son expérience du marché financier américain - Aldred avait été président du Puritan Trust de Boston - de même que ses contacts personnels, furent déterminants pour convaincre des investisseurs d'abord fort hésitants. Car le marché financier américain ne s'ouvrit pas facilement. Un projet d'émission de debentures en 1898 dut être abandonné et ce fut tant bien que mal que l'on réussit à lancer une première série d'obligations en 1900. Les choses n'allèrent pas de soi non plus en 1904, la compagnie d'électricité devant consentir des primes de remboursement importantes lors de la vente des titres ${ }^{7}$. Les difficultés persistaient encore en 1907 en raison d'une crise assez grave aux États-Unis. On eut alors l'idée de préparer l'émission de debentures destinés cette fois au marché britannique. Cet emprunt était garanti par le nantissement d'une partie des titres des filiales de la $S W P C$.

6 Dans cette partie, nous nous appuyons sur des documents d'archives de la $S W P C$, dont les procès-verbaux du conseil d'administration, les «year book» (états financiers très détaillés), etc.

\footnotetext{
Le total de ces primes s'élevait à $712100 \$$ sur un emprunt de $4287750 \$$, soit près de
} $17 \%$ contre environ $10 \%$ seulement lors de l'émission de 1900. 
Les années 1904-1907 virent cependant la situation de la compagnie d'électricité shawiniganaise s'améliorer graduellement. Dans la décennie qui suivit, elle réussit même à augmenter son capital-action qui devint ainsi la plus grande source de son financement: la valeur nominale des actions en circulation passa de 7 millions de dollars en 1909 à 11 millions de dollars en 1912. Le premier conflit mondial donna le signal d'une nouvelle vague d'augmentations de capital, de sorte qu'en 1919 les actions totalisaient 20 millions de dollars, soit $57,1 \%$ du capital investi.

La SWPC participa pleinement à la grande effervescence financière des années 1920. En plus d'accroître substantiellement ses immobilisations, la compagnie procéda entre 1927 et 1929 à l'absorption de nombreuses filiales du secteur de l'électricité et elle fusionna celles du secteur de la chimie. Ce processus intense de concentration fit passer les capitaux investis de 35 millions de dollars en 1919 à 146 millions dix ans plus tard. Pareille poussée des investissements conduisit la SWPC à recourir massivement au marché des obligations. Celles-ci en vinrent même à concurrencer les actions comme source de capitaux. Entre 1919 et 1929 , elles passèrent en effet de 9,4 millions $(28,5 \%$ des capitaux investis) à plus de 59 millions de dollars (soit $40 \%$ des capitaux investis, contre $49,5 \%$ pour le capital-action).

Les taux d'intérêt des emprunts à long terme contractés au cours des années vingt étaient relativement élevés. Dans la décennie 1900, alors que la solvabilité de la compagnie était beaucoup moins grande, ils oscillaient entre $4,5 \%$ et $5 \%$. En 1920-1921, la compagnie dut s'accommoder de taux tournant autour de $5,5 \%$ et $6 \%$. Dans une conjoncture aussi défavorable, les dirigeants préférèrent les emprunts à moyen terme («notes» d'une durée variant le plus souvent entre quatre et sept ans). Ils se gardaient la possibilité de racheter ces «notes» en lançant de nouvelles obligations lorsque la situation du marché se serait améliorée.

Cette stratégie permit à la $S W P C$ de ramener le taux d'intérêt de la plupart de ses dettes à long terme à environ $4,5 \%$ à la fin des années 1920. Ce qui passait sans doute pour une politique rapportant des profits supplémentaires à une entreprise en bonne position, s'avéra un élément crucial pour traverser la crise des années trente. En période de récession profonde, chaque point au chapitre des intérêts de la dette à long terme entraînait évidemment des déboursés de plusieurs dizaines, voire de centaines de milliers de dollars. Notons d'ailleurs que le mouvement de rachat des anciennes obligations se poursuivit jusqu'à la fin des années 1940. Il est toutefois une clause dans les termes régissant le remboursement des obligations qui fut durement ressentie par la SWPC: l'obligation presque généralisée de payer en dollars américains. Le taux de change était devenu littéralement un cauchemar pour les dirigeants de la compagnie: la SWPC perdit ainsi $378000 \$$ pour la seule année 
1933. A partir de cette époque, sauf en de rares exceptions, la compagnie prit bien soin d'émettre des titres dont le paiement du principal et des intérêts se ferait en dollars canadiens.

Confrontés à des difficultés financières constantes pendant toute la décennie, les dirigeants durent faire preuve d'imagination. En plus de pratiques comptables destinées à minimiser les pertes encourues ${ }^{8}$, la SWPC adopta des politiques visant à s'affranchir du marché financier: prélèvement sur le capital-action afin d'amortir les frais d'émission et de rappel des obligations; remboursement des anciennes séries d'obligations à taux d'intérêt élevé grâce à l'émission d'obligations à taux d'intérêt plus faible. Et surtout, on vit la part de l'autofinancement croître progressivement. Ne comptant que pour 6,9\% des capitaux investis en 1931, les bénéfices non-répartis atteignaient à nouveau le cap des $10 \%$ en 1940. On procéda aussi, de 1930 à 1938, à une série de transactions qui aboutirent à une sorte de recapitalisation de la Shawinigan Chemicals: onze ans après sa création, cette dernière en effet put rappeler l'ensemble de ses obligations en échange d'actions nouvelles.

Les tendances inscrites au cours des années de crise se prolongèrent dans la décennie suivante. Il est remarquable par exemple de constater à quel point la timidité des investissements des années trente semble vouloir s'étirer jusque dans l'immédiat après-guerre, faisant fi, jusqu'à un certain point, de la reprise pourtant réelle qui accompagna le deuxième conflit mondial. En fait, il fallut attendre 1946, et surtout 1948, pour que les capitaux investis reprennent un rythme de croissance tant soit peu vigoureux. Tout se passait comme si la $S W P C$, déjà bien pourvue en ressources énergétiques, s'était contentée de les exploiter au maximum au cours des années de guerre, quitte même à augmenter considérablement ses achats d'énergie auprès de firmes concurrentes. Enfin, la tendance à l'autofinancement se fit de plus en plus marquée. Une moyenne calculée pour les années 1948-1950 laisse entrevoir que la part des capitaux investis provenant de bénéfices non-répartis atteignait les 21\%: on avait donc doublé la part de l'autofinancement en moins de dix ans.

\section{Le groupe Wabasso 9}

Créé en 1907 et de dimensions plus modestes que les autres, ce groupe spécialisé dans le textile ne connut pas les mêmes besoins finan-

8 En 1931, par exemple, on amortit la «réserve capitale» créée lors de l'absorption de la Laurentide et de la St.Maurice Power en 1927, laquelle était à toutes fins pratiques une sorte de surplus d'apport. Cela eut comme effet de rendre beaucoup moins évidente la baisse sensible de la valeur du portefeuille, notamment du côté de la chimie.

9 Les informations sur ce groupe proviennent des rapports annuels tels que publiés dans Annual Financial Review, et de P. Lanthier et A. Gamelin, L'industrialisation de la Mauricie: dossier statistique et chronologique, 1870-1975 (Trois-Rivières, Groupe de Recherche sur la Mauricie, 1981), 426-460. 
ciers. Tout d'abord, les liquidités y tenaient une place plus importante. Ensuite, la faiblesse des investissements l'éloignait du marché des titres. Ainsi, de 1750 000\$ au départ, le capital-action n'augmenta qu'une seule fois, en 1927-1929, pour atteindre $4192000 \$$. En 1939, il fut même réduit à 2 millions afin d'amortir des biens immobiliers et les primes sur obligations. Par la suite, il ne bougea pas. La petitesse du capital joua d'ailleurs un rôle décisif au niveau de la propriété, car elle permettait aux principaux actionnaires de contrôler la société avec peu. A cela s'ajoutaient des investissements croisés entre la maison mère et ses filiales, ayant pour but une protection mutuelle. En 1930, par exemple, la St.Maurice Valley Cotton possédait une part substantielle du capital de la Wabasso: $594052 \$$ sur un total de 4,2 millions, soit $14 \%$.

L'analyse du passif révèle aussi un recours assez limité au marché des obligations. La compagnie avait fait l'émission dès 1907 de $981000 \$$ à $6 \%$, auxquels vinrent s'ajouter $750000 \$$ à $7 \%$ en 1922 . Parallèlement, les filiales de la société, la Shawinigan Cotton et la St.Maurice Valley, avaient émis respectivement 2,5 millions en 1909 et 1,5 millions en 1912 (porté à 2,5 en 1923). En 1936 enfin, en vue d'alléger le paiement des intérêts, la société remboursa les obligations en circulation grâce à une émission de trois millions à des taux d'intérêt plus faibles: 3,5\% (remboursable en 1941), 4\% (1948) et 4,5\% (1951). En 1950 , toutes les obligations du groupe avaient été dûment amorties, ce qui renforçait la situation financière de la société.

En fait, la stratégie principale de la société consista à cumuler au fil des années du profit non distribué dans le but de préserver son fonds de roulement et, le cas échéant, d'acquérir des immobilisations d'importance moyenne (comme deux génératrices à vapeur en 1941). Cette pratique mit petit à petit la société à l'abri d'intervenants extérieurs. Bien qu'elle ait eu accès dès 1907 au marché financier, elle ne parvint que lentement à jouir d'un fonds de roulement adéquat. Les dirigeants durent d'abord faire appel à d'autres sources. Par exemple, en 1910, la Wabasso bénéficia d'une subvention de $25000 \$$ de la municipalité de Trois-Rivières et d'exemptions de taxes municipales pour 20 ans. Mais l'expédient le plus important fut le crédit à court terme. Ainsi, en 1917, la Shawinigan Cotton emprunta $109666 \$$ auprès de la Banque d' Hochelaga. Dans l'entre-deux-guerres, la Wabasso bénéficia de prêts bancaires qui s'élevèrent jusqu'à $1036000 \$$ en 1929 et 1930 . Ils étaient garantis par des debentures de $600000 \$$ à $6 \%$ (autorisées mais non émises) de 1916 à 1921, puis par ses propres obligations à partir de 1922, ou même par du coton brut, comme en 1920 et en 1924 . La Wabasso pouvait encore obtenir du crédit en hypothéquant une partie de ses propriétés, mais pour de petits montants seulement, comme ce fut le cas de 1919 à 1922 (19 000\$); ou mieux, recevoir des prêts de sa filiale, la St.Maurice Valley Cotton: $131000 \$$ en 1923 et $484646 \$$ l'année suivante. Après 1934, la société cessa ses emprunts bancaires. 
Grâce à sa politique d'autofinancement, elle put se suffire à elle-même et parvint à s'entendre avec ses fournisseurs en coton et en machinerie pour obtenir des avances.

Bref, après une période d'expansion qui nécessita le recours au marché financier et à des emprunts à court terme, la société ralentit son expansion, remboursa ses dettes et reposa ses assises sur un autofinancement cumulatif.

\section{La Consolidated Paper et ses ancêtres ${ }^{10}$}

L'industrie des pâtes et papiers rencontrait, sur le plan financier, des problèmes tenant à la fois à son développement et à la conjoncture. En effet, si l'industrie sut toujours pourvoir à ses besoins en liquidité grâce à l'autofinancement, en revanche, la croissance des immobilisations, en particulier pour la fabrication du papier, exigeait du capital extérieur. Cependant, entre 1910 et 1925, époque où le plus gros de ce développement eut lieu, le marché obligataire n'offrait pas de taux d'intérêt avantageux pour les compagnies. Bien qu'il fallut recourir aux emprunts à long terme, l'on chercha dans la mesure du possible à ne pas trop en dépendre et à varier les sources de financement. C'est ce que montre l'examen des cas de la Laurentide et de la Wayagamack.

\section{a - La Laurentide Pulp Co}

Cette société, établie à Grand-Mère depuis 1887, connut deux générations de propriétaires. Une première, animée par les Américains A. Pagenstecher et W. Curtis, attira des investisseurs germano-américains qui souscrivirent non seulement au quart des actions, mais à une émission d'obligations de $100000 \$$. La seconde génération arriva en 1896, quand Pagenstecher et Curtis vendirent leurs intérêts au général américain Russell $\mathrm{A}$. Alger et à des hommes d'affaires canadiens gravitant autour du CPR et de la Banque de Montréal. Alger conserva une part importante dans la société jusqu'en 1907, après quoi il se retira et le contrôle de la Laurentide devint avant tout canadien. Bien que quelques actionnaires parvinrent à y maintenir une certaine prépondérance, la tendance était dès lors à la dispersion: à son absorption en 1927, la société comptait près de 3000 actionnaires. Parmi ceux-ci, les

10 Pour cette partie, de nombreux éléments ont été tirés de: Annual Financial Review (rapports annuels des sociétés concernées); A. Lafrance, Histoire d' une compagnie papetière au Québec: "La Belgo» (1900-1925), Thèse de maîtrise (sociologie), Université du Québec à Montréal, 1976; J. Niosi, «La Laurentide (1887-1928): pionnière du papier-journal au Canada», Revue d'histoire de l'Amérique française, 29,3 (décembre 1975): 375-415; R. Parent, Histoire économique et sociale de la Wayagamack Pulp and Paper Co. (1910-1929), Thèse de maîtrise, Université du Québec à Montréal, 1975; G. Piédalue, «Les groupes financiers et la guerre du papier au Canada, 1920-1930», Revue d' histoire de l'Amérique française, 30,2 (septembre 1976): 223258; supplément de Forêt et Conservation à l'occasion du $50 \mathrm{e}$ anniversaire de la ConsolidatedBathurst Inc., mai 1982. 
maisons de courtage (comme la McDougall \& McDougall) occupaient un espace de plus en plus grand.

Forte de ces appuis, la société n'eut aucune difficulté à financer ses immobilisations. Le recours, après 1896, aux obligations ne fut d'ailleurs pas énorme: une seule émission, en 1900, de $1200000 \$$. Par la suite, la montée des taux d'intérêt au-delà des $6 \%$ diminua l'attrait pour cette source de capitaux. Ses contacts avec le monde de la banque l'incita à faire de gros emprunts à court terme en 1913-1915 et en 19201926 pour accroître ses immobilisations. La première vague atteignit son point culminant en 1915, avec 2037 437\$; et la seconde en 1921, avec plus de 5 millions. Celle-ci fut assurée exclusivement par la Banque de Montréal, dont les dirigeants figuraient parmi les actionnaires importants de la Laurentide. Ces emprunts exigeaient cependant de solides gages. C'est pourquoi la société dut au préalable actualiser la valeur de ses actifs une première fois en 1911 et une seconde en $1920^{11}$. En somme, sous les apparences d'une politique conservatrice, la société donnait au capital bancaire un rôle important dans le financement global de ses actifs. Quant au fonds de roulement, il était assuré par un autofinancement fort appréciable: en 1927, même après deux actualisations, il comptait pour $18 \%$ des capitaux investis.

\section{b - La Wayagamack}

La Wayagamack offrait de nombreuses similitudes avec la Laurentide: tout d'abord, elle fut lancée en 1911 par des Canadiens ayant d'importants contacts personnels du côté des chemins de fer et des banques. Ensuite, l'évolution des actionnaires était comparable à bien des égards: départ ou baisse de l'influence des fondateurs, et leur remplacement par des maisons de courtage. En 1927, celles-ci possédaient $59,1 \%$ des actions de la Wayagamack. Mais, à la différence de la Laurentide, on ne peut pas parler sérieusement de dispersion: le nombre d'actionnaires, de 1917 à 1924, gravitait entre 200 et 250. Cette situation, à vrai dire, ne surprend guère, car, de 1911 à 1927, il n'y eut pas d'augmentation de capital. Et encore, en 1927, on ne procéda qu'à une distribution gratuite d'actions et, l'année suivante, à une modeste augmentation de deux millions.

Les emprunts procurèrent à la compagnie l'autre part importante de son financement. Pour trouver des prêteurs, elle fit appel à la Quebec Saving Trust, société dirigée par l'un de ses administrateurs. Cette société plaça ainsi une émission d'obligations à $6 \%$ appelée par tranches suc-

11 Par actualisation, on entend cette opération qui consiste: 1 - à réinclure dans la valeur des actifs la part de financement de certaines sources qui n'apparaissent plus au bilan, comme les obligations remboursées et certaines réserves ou provisions; 2 - à tenir compte des effets de la variation des prix sur la valeur des immobilisations. L'actualisation se fait généralement en augmentant le capital par distribution gratuite d'actions aux actionnaires. 
cessives de 1912 à 1922. Elle eut également à gérer d'importants emprunts bancaires en 1913-1915 et surtout en 1920-1922 (près de 2 millions de dollars en 1921). Signalons qu'en 1921, l'emprunt à court terme était gagé par les propriétés forestières de la société et l'émission d'une tranche d'obligations. Ici aussi la taille importante de l'entreprise devenait un atout majeur de solvabilité. Quant à l'autofinancement, il progressait régulièrement. Sa supériorité apparente sur celui de la Laurentide (voir tableau 1) ne doit pas faire illusion, compte tenu des réorganisations comptables de celle-ci.

Dans les deux cas, se précise une évolution nette vers un financement plus «institutionnel», que ce soit par l'intermédiaire d'émissions de titres ou par l'intervention de maisons de courtage. Au terme de cette évolution, les conditions étaient réunies pour réaliser la gigantesque fusion qui mena, de 1926 à 1932, à la formation de la Consolidated Paper Co Ltd.

\section{c - La Consolidated Paper Co Ltd}

Sans reprendre en détail la genèse du regroupement, il est nécessaire d'en résumer les grandes lignes du point de vue financier. Tout d'abord, la St.Maurice Valley Corp, en 1925, qui unifiait les actifs de la Belgo Pulp \& Paper et de la St.Maurice Paper, en reprenait également les titres, tout en bénéficiant de prêts de 2 millions de dollars de la Banque Royale et d'émissions supplémentaires d'obligations. Puis la Canada Power \& Paper $(C P \& P)$, qui fusionnait les intérêts de la St.Maurice Valley et de la Laurentide, emprunta $3175000 \$$ de la Banque Royale et émit pour 28 millions d'obligations. Semblable scénario se répéta lorsque la Wayagamack, la Port Alfred et l'Anglo-Canadian Pulp \& Paper furent absorbées. De sorte qu'en 1930, le capital investi de la $C P \& P$ se chiffrait à 213 millions de dollars, se répartissant comme suit: $24,5 \%$ en actions, $44,5 \%$ en obligations et debentures, $7,1 \%$ en prêts bancaires, $18,6 \%$ en réserves et surplus, et $5,3 \%$ en créditeurs divers. Le montant était colossal, tout comme la dépendance à l'endroit des institutions financières. Le CPR et la Sun Life figuraient parmi les plus importants obligataires de la société.

La crise fit craquer ce gros édifice. Les créanciers de la société formèrent un comité afin de rééquilibrer les finances. Ce comité se trouvait sous l'influence du CPR, de la Banque de Montréal et de la Banque Royale. Le capital-actions fut considérablement réduit (tombant à 1,4 millions), et les obligations ramenées à près de 53 millions, tandis que les prêts bancaires conservèrent la même valeur $(15,1$ millions). Le comité décida en outre que ces derniers seraient remboursés en priorité, même avant les obligations.

De fait, en 1940, la société s'était entièrement acquittée de ses dettes à l'endroit des banques, et dix ans après, plus de la moitié de ses 
obligations avaient été amorties. En 1947, la société avait même réussi à remplacer ses obligations à 5,5\% de 1932 par de nouvelles à 3,5\%. D'autre part, l'autofinancement de la société avait atteint un niveau fort important (près de 68\% du capital investi en 1948-1950), tandis que les actions, qui n'avaient été augmentées qu'à cinq millions en 1941, conservaient une part modeste. La société était à nouveau solvable, en dépit des difficultés rencontrées avec le groupe Hearst qui ne pouvait plus rembourser ses dettes. Celles-ci, évaluées à $1500000 \$$ en 1944, furent transformées en «notes» de 10 ans à 3\%. Enfin, en 1946, la distribution des dividendes redevint réalité. La société avait donc surmonté ses embarras de remarquable façon.

L'analyse individuelle des groupes permet de dessiner la personnalité financière de chacune. La Wabasso a déployé un éventail très diversifié de sources de financement à court terme. L'intervention fréquente des milieux bancaires a marqué l'histoire de la Consolidated Paper et de ses ancêtres. Et les capitaux étrangers, notamment par les emprunts à long terme, ont soutenu la $S W P C$ plus que les autres. Dans une certaine mesure, les préférences dans les sources de financement exprimaient les liaisons fonctionnelles entre les groupements d'intérêt et l'entreprise. Par exemple, pour assurer le financement d'une partie des immobilisations, la Wayagamack avait eu recours aux obligations, tandis que la Laurentide, très proche de la Banque de Montréal, avait privilégié l'emprunt bancaire. Cette marge de manoeuvre toutefois, a été rendue possible du fait que les entreprises de pâtes et papiers généraient un autofinancement très important permettant d'amortir rapidement les emprunts à court terme. Une entreprise hydro-électrique dont l'autofinancement était proportionnellement moindre n'aurait pas indéfiniment adopté pareille voie de financement. Ces variantes individuelles s'inscrivaient dans un processus de maturation des activités. La Wabasso, société textile, avait de plus en plus des préoccupations de liquidités qu'il aurait été peu avantageux de soutenir avec du crédit à long terme. La SWPC devait quant à elle financer d'importantes immobilisations, et la stabilité de son marché rendait intéressant le recours aux obligations ${ }^{12}$. Au fur et à mesure que les sociétés vieillissaient, les considérations d'ordre sectoriel devenaient déterminantes dans le choix des sources de financement.

\section{$C$ - Évolution des stratégies de financement}

En dépit des particularités propres à chaque groupe, l'analyse révèle une similitude de politique selon les époques considérées. Il est possible

12 Dans l'entre-deux-guerres, les grandes entreprises françaises de production et de distribution d'énergie électrique avaient fait le même choix dans les sources de financement: les émissions d'obligations devinrent plus importantes que les augmentations de capital. A ce sujet, on se reportera à la thèse en cours de rédaction de $\mathrm{P}$. Lanthier, Le rôle des groupes industriels internationaux dans l'électrification de la France, 1880-1940. (Université de Paris X-Nanterre). 


\section{TABLEAU 1}

Structure du passif des trois groupes industriels

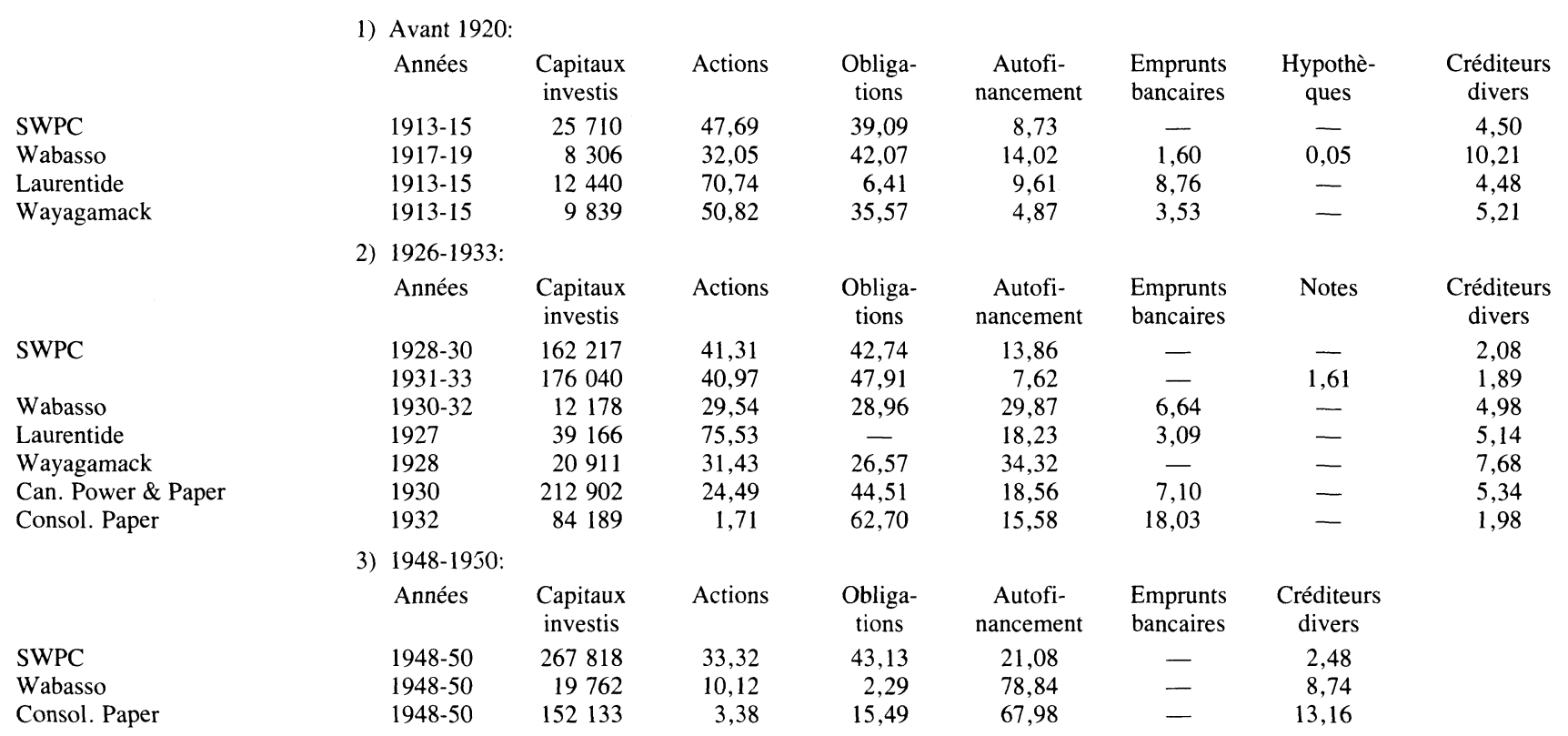

Source: Rapports annuels

Les capitaux investis sont en milliers de dollars courants, tandis que les principaux postes du passif sont exprimés en pourcentages. On notera que le poste «Autofinancement» comprend aussi bien l'autofinancement obtenu par bénéfices non distribués que celui accumulé par remboursement des emprunts à long terme. 
en effet de distinguer trois grandes périodes dans les attitudes financières: les débuts, les années dix et vingt, et les années trente et quarante. Le tableau 1 présente la structure des passifs des trois groupes à la fin de ces périodes.

\section{1 - Les débuts}

Avant la guerre de 1914-1918, la Mauricie était une région certes riche en ressources naturelles, mais dépourvue sur le plan financier. Il ne faut donc pas s'étonner que les groupes aient connu initialement de nombreuses difficultés à s'approvisionner en capitaux. Deux voies furent alors choisies. Tout d'abord le primat des relations individuelles. Avant la guerre, les contacts personnels entre les administrateurs et les investisseurs furent décisifs. Qu'il s'agisse des rapports entre Pagenstecher et les milieux germano-américains, Aldred et les financiers américains, ou des administrateurs communs de la Banque de Montréal et de la Laurentide, tous confirment le rôle crucial des individus et de l'étendue de leurs liaisons d'affaires. On notera d'ailleurs l'importance, pour cette époque, du capital et des administrateurs étrangers. L'ensemble de ce phénomène n'était nullement propre à la Mauricie. D. G. Paterson a observé quelque chose d'analogue en Colombie Britannique, et Lance Davis a noté le même processus aux États-Unis: en l'absence de banques susceptibles d'exercer un pouvoir à l'échelle nationale, les liens directs entre pourvoyeurs de fonds et entrepreneurs étaient déterminants avant $1920^{13}$. Au Canada, il fallait du capital «symbolique» pour lancer une entreprise de grande échelle en milieu périphérique: des hommes prestigieux (Greenshields, Forget) ou ayant déjà une forte réputation sur le plan des affaires (Pagenstecher, Van Horne), ou de nombreuses connaissances dans les cercles capitalistes.

Plus important encore, le recours aux emprunts. Dès le départ, il fallait se procurer des avances ou des prêts à court terme afin d'obtenir des liquidités adéquates ou même financer certaines immobilisations. Mais pour obtenir ces prêts, on devait bénéficier de solides garanties. La présence de grandes usines et de machinerie lourde servit à cet effet: on pouvait les offrir en gage pour d'éventuels emprunts (Wayagamack, $S W P C$ ), ou même pour hypothéquer (Wabasso). Dans ce processus, et ceci est également valable pour la période suivante, le prêt bancaire prenait une place de tout premier plan. La montée du taux d'intérêt des obligations à plus de $6 \%$ dès avant 1920 , pour les compagnies de pâtes et papiers et pour la Wabasso, les incitèrent à avoir recours plus tôt et plus systématiquement que la $S W P C$ aux emprunts bancaires. Si la banque n'intervint pas à titre de gros actionnaire dans les entreprises,

13 D. G. Paterson, «European Financial Capital and British Columbia: an Essay on the Role of the Regional Entrepreneur», BC Studies, 21 (Spring 1974): 33-47. L. Davis, «The Capital Market and Industrial Concentration in the US and UK, a Comparative Study», Economic History Review, 2nd series, 19 (1966). 
en tout cas joua-t-elle un rôle décisif dans le domaine des prêts à court terme.

\section{2 - Les années dix et vingt}

Les stratégies employées avant 1914 aboutirent à d'excellents résultats pour la plupart des grandes sociétés mauriciennes. Et le succès ouvrit de nouvelles possibilités. Il devenait en effet de plus en plus facile d'avoir accès aux capitaux de Montréal et des États-Unis. L'on pouvait désormais «dépersonnaliser» les contacts financiers. Le nombre d'actionnaires des grandes sociétés grossit considérablement. Ainsi, pour la $S W P C$, il passa de 2000 en 1909 à plus de 17000 en 1930. A cette époque, d'ailleurs, les actions de plusieurs grandes sociétés industrielles oeuvrant en Mauricie se canadianisèrent ${ }^{14}$. Par contre, au niveau des obligations et des actions privilégiées, le recours à l'étranger non seulement persista, mais s'accrut. La Canada Pulp \& Paper possédait de nombreux obligataires en Grande-Bretagne et actionnaires privilégiés aux États-Unis. La SWPC, comme on l'a vu, écoula beaucoup de ses obligations aux États-Unis.

D'autre part, la machine financière s'institutionnalisait, en particulier pour les entreprises de grande taille. Du côté des pâtes et papiers, l'arrivée des maisons de courtage est frappante. On peut penser qu'elles avaient hérité des actions que ne pouvait pas souscrire le capital bancaire $^{15}$. Mais les banques n'avaient pas disparu du paysage. Bien au contraire: l'instabilité économique, notamment en 1921-1922, rendait nécessaires de très gros emprunts bancaires ou l'émission de «notes».

Après 1926, la baisse des taux d'intérêt incita les entreprises à se concentrer et à émettre un montant énorme de titres, en grande partie des obligations. En 1930, le capital investi de la SWPC était de plus de 184 millions de dollars et celui de la Canada $P \& P$ de 213 millions. Même la Wabasso avait suivi le mouvement et se risqua à augmenter son capital et à faire des emprunts bancaires. Cette politique n'était toutefois pas sans aléa. Car elle mettait encore plus qu'auparavant les groupes à la merci de la conjoncture financière. Mais, tant que le marché resta favorable, la Mauricie en profita. Après 1930, il n'en fut plus de même.

\section{3 - Les années trente et quarante}

La crise fit prendre conscience du danger qu'il y avait de dépendre trop exclusivement du marché financier. En plus de provoquer d'im-

\footnotetext{
14 Mentionnons de plus que les entreprises lancées au cours de cette période étaient dans bien des cas canadiennes dès le départ. C'était le cas par exemple de la Wayagamack et de la St.Lawrence Paper. Voir P. Lanthier et A. Gamelin, L'industrialisation de la Mauricie, dossier statistique et chronologique (Trois-Rivières, Groupe de recherche sur la Mauricie, 1981).

La loi sur les banques au Canada interdisait en effet à celles-ci de détenir plus de $10 \%$ des actions de sociétés non bancaires. Voir à ce sujet: J. Niosi, Le contrôle financier du capitalisme canadien (Montréal, Presses de l'Université du Québec, 1978).
} 
portantes dévaluations du capital investi en 1930-1931, elle incita les groupes à recourir à de nouvelles stratégies.

Tout d'abord, on chercha, dans la mesure du possible, à atténuer cette dépendance. Par exemple, en 1938-1939, la Wabasso et la SWPC réduisirent leurs capitaux notamment pour amortir la charge financière constituée par les primes sur obligations. Mieux, après 1940, toutes les sociétés émirent de nombreuses obligations non pas pour investir, mais pour bénéficier de la baisse des taux d'intérêt. De 6\% dans les années vingt, ces taux étaient tombés entre 3,5 et 4\% après 1935-1940. De son côté, la SWPC fit «canadianiser» le paiement de ses obligations, évitant ainsi de grosses pertes sur les variations du taux de change.

Mais plus généralement, on privilégia l'autofinancement soit en grossissant la part des bénéfices non distribués, soit en remboursant les dettes à moyen et à long terme. La période, pour les trois groupes, en fut une d'assainissement financier et il en résulta très peu d'investissements majeurs. Le fonds de roulement en bénéficia, car l'autofinancement se substitua aux emprunts bancaires dans l'obtention de liquidités. Des ententes furent même conclues avec les clients et les fournisseurs afin d'obtenir des avances à court terme ou de meilleures modalités de paiement: la Wabasso, en particulier, utilisa cette méthode, ainsi que la $S W P C$ avec des passifs différés en 1949-1950, et la Consolidated Paper avec les «notes» du groupe Hearst en 1944. L'autonomie financière était à l'ordre du jour. La Wabasso et la Consolidated, grâce à cette politique, purent relancer non seulement leur fonds de roulement, mais aussi leurs investissements dans de petites immobilisations en Mauricie. Dans la mesure du possible, enfin, on évitait les augmentations de capital. Seule la SWPC en fit une de 20 millions de dollars en 1947, dans le but de construire de nouvelles centrales.

Cependant, cette reprise ne déboucha pas sur une expansion des groupes en Mauricie. Certes, la SWPC érigea une dernière centrale dans les années cinquante (Beaumont), mais elle porta en même temps une partie considérable de son développement en dehors de la région: par exemple, l'absorption de la Southern Canada en 1957 et des investissements en pétrochimie dans la région montréalaise. Du côté de la Consolidated Paper, il y eut expansion (par exemple à Pontiac en 1964), mais pas en Mauricie; en outre, une bonne partie de son développement privilégia l'acquisition d'actifs déjà existants au détriment de nouvelles constructions. Et la Wabasso, absorbée en 1955 par le groupe Woods de Toronto, construisit très peu en région. En somme, à partir des années cinquante, la croissance ne se fit pas exclusivement à coups d'émissions de titres, mais aussi par fusion, acquisition d'actifs, ou encore par association dans un projet commun, ce qui constituait une façon de se développer sans avoir recours au marché financier. Ce processus ne favorisa pas la Mauricie. 


\section{CONCLUSION}

Mettre en relation financement et développement régional ne va pas sans quelques difficultés d'interprétation, surtout lorsque les capitaux locaux ne constituent pas une base d'accumulation suffisante, et que la région se trouve mise en valeur par des entrepreneurs venus de l'extérieur. Il existe des liens entre les deux, mais ils ne s'expriment pas de façon simple.

Tout d'abord, en ce qui concerne le plafonnement industriel des années cinquante, il serait nettement exagéré de l'expliquer par des raisons exclusivement financières. D'autres facteurs, variés et plus importants, sont intervenus dans ce processus. Tout au plus, le désir de se rendre aussi indépendant que possible des capitaux extérieurs, manifeste après 1930, peut-il être en partie responsable de la croissance des groupes par acquisition d'actifs en dehors de la Mauricie.

Mais l'analyse n'a pas écarté tout rapport entre financement et croissance économique régionale, loin de là. On peut en effet avancer certains schémas explicatifs et les proposer à titre d'hypothèses dans le cadre d'une étude élargie ou comparative. L'implantation de grandes sociétés dans un espace économique encore peu développé et à faible densité démographique posait des difficultés majeures. L'éloignement des marchés, la nécessité de tout construire à neuf, d'investir dans des infrastructures comme les transports et les communications, qui n'étaient pas du ressort direct de la société mais dont elles dépendaient de façon vitale, suscitaient bien des hésitations, voire la méfiance des bailleurs de fonds. C'est sans doute ce qui explique qu'au tout début du siècle, les firmes étudiées n'avaient pu bénéficier pleinement de la conjoncture expansionniste de l'époque. Pour forcer ce blocage, elles mirent de l'avant une même stratégie: profiter de contacts personnels avec quelques grands noms du milieu financier et mettre en gage une partie de leur patrimoine (grandes usines, immenses domaines forestiers, etc.) afin d'obtenir d'importants crédits à court terme. Par la suite, le développement d'institutions spécialisées permettant le drainage d'un grand nombre de capitaux isolés, de même que la maturation de firmes désormais beaucoup plus solvables, entraînèrent une «dépersonnalisation» progressive du financement. Ainsi libérées des contraintes initiales, les sociétés industrielles étudiées orientèrent leurs stratégies selon des modalités propres à chaque secteur d'activité. Cet affranchissement, doublé d'un élargissement de l'éventail des stratégies, fit en sorte qu'au cours des années 1910-1920, l'histoire financière de ces sociétés se trouva de plus en plus fortement et directement rythmée par la conjoncture internationale.

On a vu aussi que la décennie 1930 a marqué un temps d'arrêt des grands investissements en région et que l'on chercha désormais à moins dépendre du marché financier. Entre 1930 et 1950, la part de l'autofi- 
nancement dans les capitaux investis ne cessa de croître, et ce dans l'ensemble des firmes. Il faut toutefois être prudent lorsque l'on veut expliquer ce mouvement. En effet, un recours aussi massif à l'autofinancement peut être interprété au moins de deux façons. Il peut s'agir soit d'une gestion conservatrice et peu expansionniste de la société, soit d'une politique d'autonomie financière résultant d'une amélioration de la position de la firme sur les marchés de consommation. A notre avis, la Wabasso appartient avant tout au premier type de société, alors que la Consolidated Power et la SWPC relèvent davantage du deuxième groupe. Dans un cas comme dans l'autre, la prudence était de mise.

Certains phénomènes significatifs se trouvant plus ou moins en marge de notre objet d'étude principal mériteraient qu'on s'y attarde un peu plus. C'est le cas de la "canadianisation» des titres qui semble s'amorcer dès les années 1910 et qui touche plus fortement le capitalactions que les obligations. Il est trop tôt pour apporter une explication valable à ce décalage. Une chose semble relativement claire cependant: d'importantes sommes furent investies par des entrepreneurs et des financiers canadiens lors de la création des grandes sociétés mauriciennes. Et s'il apparaît évident qu'ils n'ont pu suffire à la tâche, force nous est de rejeter l'hypothèse d'une hostilité des milieux d'affaires canadiens aux défis posés par la mise sur pied de telles entreprises au début du siècle. Par ailleurs, certains résultats des études de cas montrent qu'il faut être particulièrement prudent lorsque l'on aborde le financement par le seul biais du contrôle. Il ne s'agit pas ici de minimiser cet aspect de la réalité économique contemporaine. Nos recherches ont toutefois permis de constater que les motivations de pouvoir des entrepreneurs n'étaient pas toujours à l'origine des mouvements visant à recapitaliser ou absorber des sociétés. Dans de nombreux cas, elles relevaient davantage de la nécessité d'ajuster le financement aux virtualités de la conjoncture économique et des marchés. Certaines stratégies jusqu'ici interprétées comme des constantes immuables des rapports de pouvoir entre groupements d'intérêt, n'appartiennent-elles pas en réalité à des moments précis de l'évolution du capitalisme industriel. 